\title{
Relationships of the platelet aggregate ratio to serum cholesterol concentration, smoking and age
}

\author{
JAMES W. DAVIS \\ M.D., F.A.C.P. \\ Phyllis E. Phillips \\ H. DANIEL LEWIS JR \\ M.D., F.A.C.P. \\ ReBecCa F. Davis
}

Veterans Administration Medical Center, Kansas City, Missouri, and the Department of Medicine, University of Kansas School of Medicine, Kansas City, Kansas

\begin{abstract}
Summary
The platelet aggregate ratio has been found to be decreased in some patients with vascular diseases suggesting the presence of increased circulating platelet aggregates. It has also been reported that hypercholesterolaemia is associated with an enhanced response of platelets to aggregating agents in plateletrich plasma. The primary purpose of this investigation was to determine correlation of the platelet aggregate ratio with the serum cholesterol concentration of men with vascular diseases. For 52 men referred because of known or suspected coronary artery disease, cerebrovascular disease, or venous thromboembolism, the correlation coefficient of 0.06 suggested that the serum cholesterol concentration within the range observed (135-360 $\mathrm{mg} / \mathrm{dl}$ ) was not a factor influencing the platelet aggregate ratio. There was not a statistically significant difference between the mean platelet aggregate ratios or the mean serum cholesterol concentrations of the 21 non-smokers and the 31 smokers studied. A correlation coefficient of 0.03 between the platelet aggregate ratio and age of the patient suggested that the platelet aggregate ratio was independent of age in men with occlusive vascular diseases.
\end{abstract}

\section{Introduction}

$\mathrm{Wu}$ and his colleagues described the platelet aggregate ratio as an index of circulating platelet aggregates and reported it to be decreased in some patients with occlusive vascular diseases ( $\mathrm{Wu}$ and Hoak, 1974, 1975; Wu, Barnes and Hoak, 1976). Their method is based on the ratio of the platelet count of platelet-rich plasma prepared from blood mixed with a solution of EDTA and formaldehyde

Requests for reprints: Dr James W. Davis, Medical Service, Veterans Administration Medical Center, 4801 Linwood Boulevard, Kansas City, MO 64128, U.S.A. to that of platelet-rich plasma prepared in the same manner except for the absence of formaldehyde. They theorized that any platelet aggregates present in blood would be fixed when withdrawn into a solution containing formaldehyde and EDTA and break apart when withdrawn into a solution containing EDTA without formaldehyde. If plateles aggregates were present in blood, the platelet counes should be lower in the platelet-rich plasma containing formaldehyde because platelet aggregates would bet spun down with erythrocytes. The observation of $\mathrm{Wu}$ and Hoak (1974) that the platelet aggregate ratio of rabbits decreased after the i.v. infusion of thrombin, suggested that this ratio monitored the presence of platelet aggregates circulating in vivo. However, Rohrer et al. (1978a) found that the platelet aggregate ratio of normal human venous blood samples varied with the rate of blood flow into the needle, suggesting that platelet aggregates formed in the needle and tubing may affect the platelet aggregate ratio. Subsequently, the platelet aggregate ratio was reported to be highly correlated with $\beta$-thromboglobulin (Chen et al., 1979) and to decrease in association with an increase in the plasma concentration of platelet factor 4 after exhaustive exercise (Levine et al., 1979). This suggests a relationship of the platelet aggregate ratio to secretion of the contents of $\alpha$-granules from platelets (Kaplan et al., 1979). The observation in patients with ischaemic heart disease of a high correlation between the platelet aggregate ratio and the platelet half-life in vivo is evidence supporting the interpretation of the platelet aggregate ratio as an index of circulating platelet aggregates in such patients (Salem, Koutts and Firkin, 1980).

The authors reviewed the records of men with vascular disease whose platelet aggregate ratios had been determined in their laboratory to learn if the 
values were related to their serum cholesterol concentration, to whether or not they smoked tobacco or to their age.

\section{Patients and methods}

The subjects of this investigation were 52 men ranging in age from 33 to 86 years (mean age, 56 years) whose platelet aggregate ratios were determined in the laboratory after referral because of known or suspected coronary artery disease, cerebrovascular disease or venous thromboembolism. They had not taken anticoagulants, anti-inflammatory agents, diazepam, dipyridamole, phenothiazines or tricyclic antidepressants during the week before testing the platelet aggregate ratio. Fasting serum cholesterol values, dated within 3 months of the platelet aggregate ratio, were obtained from the hospital records after determination in the clinical chemistry laboratory by an automated enzymatic technique.

Platelet aggregate ratios were determined essentially as described by Wu (1978). A first sample of antecubital venous blood was drawn into a plastic syringe containing a buffered solution of EDTA and formaldehyde and a second sample into another syringe containing a buffered solution of EDTA without formaldehyde. The contents of the syringes were immediately mixed by inverting 3 times, then transferred to siliconized glass tubes and incubated at room temperature for $15 \mathrm{~min}$ before centrifugation at $220 \mathrm{~g}$ (bottom of tube) for $8 \mathrm{~min}$. The supernatants were diluted $1: 20$ in $1 \%$ ammonium oxalate for platelet counting by phase-contrast microscopy using duplicate pipettes. The ratio of the platelet count in the supernatant containing formaldehyde to that without formaldehyde was calculated.

The significance of the differences between mean platelet aggregate ratios and mean serum cholesterol concentrations was determined by the Wilcoxon rank sum test. The Spearman rank correlation coefficient was used to determine the association of the platelet aggregate ratio with age and serum cholesterol concentration.

\section{Results}

The fasting serum cholesterol concentrations of the 52 patients studied ranged from 135 to $360 \mathrm{mg} / \mathrm{dl}$. The mean platelet aggregate ratios $( \pm$ s.d.) of the 26 men with the lowest serum cholesterol concentrations $(135-225 \mathrm{mg} / \mathrm{dl})$ and of the 26 men with the highest serum cholesterol concentrations (230-360 $\mathrm{mg} / \mathrm{dl})$ were $0 \cdot 79 \pm 0 \cdot 17$ and $0 \cdot 78 \pm 0 \cdot 13$ respectively $(P>0 \cdot 1)$. The platelet aggregate ratio correlated poorly with the fasting serum cholesterol concentration $(r=0.06 ; P=0.7)$ and with the age $(r=0.03$; $P>0.9$ ) of the 52 men.

The subjects included 31 habitual smokers of tobacco products (mostly cigarettes) and 21 nonsmokers. The mean platelet aggregate ratio ( \pm s.d.) of the smokers was $0.76 \pm 0.16$ and of the nonsmokers was $0 \cdot 81 \pm 0 \cdot 14(P>0 \cdot 1)$. The mean fasting serum cholesterol ( \pm s.d.) of the 31 smokers (whose mean age was 55 years) was $227 \pm 52 \mathrm{mg} / \mathrm{dl}$ and of the 21 non-smokers (whose mean age was 58 years) was $243 \pm 52 \mathrm{mg} / \mathrm{dl}(P>0 \cdot 1)$.

\section{Discussion}

It has been reported (Carvalho, Colman and Lees, 1974) that the platelets of patients with type II hyperlipoproteinaemia and a mean serum cholesterol concentration of $354 \mathrm{mg} / \mathrm{dl}$ had an enhanced aggregation response to epinephrine, ADP or collagen in platelet-rich plasma. Although Wu et al. (1975) described increased circulating platelet aggregates in Rhesus monkeys with experimentallyinduced hypercholesterolaemia, they found a poor correlation between the platelet aggregate ratio and the serum cholesterol concentration. The low correlation coefficient $(0.06)$ between the platelet aggregate ratio and the serum cholesterol concentration of the present patients suggests that the serum cholesterol concentration within the range observed $(135-360 \mathrm{mg} / \mathrm{dl})$ is not a major factor influencing the platelet aggregate ratio in man.

Although in 2 studies before 1960 (Thomas, 1958; Karvonen et al., 1959) men who smoked tobacco had higher serum concentrations of total cholesterol than did non-smokers, results are in agreement with those of 2 more recent studies (Pozner and Billimoria, 1970; Enger et al., 1977) which showed the mean serum cholesterol concentration of male smokers to be no higher than that of non-smokers.

Erikssen, Hellem and Stormorken (1977) found a small but highly statistically significant increase in 'platelet adhesiveness' in smokers as compared with non-smokers when the former were tested at least $12 \mathrm{hr}$ after the last cigarette was smoked. After finding that the mean platelet aggregate ratios of 2 groups of healthy volunteers decreased during a 20-min period in which 2 cigarettes were smoked (Davis and Davis, 1979, 1980), the authors analysed the present series of 52 men to see whether the 31 habitual smokers had lower ratios than did the 21 non-smokers. This was found to be the case, but the difference was not statistically significant. Rohrer et al. (1978b) found a lower mean platelet aggregate ratio in normal smokers than in normal non-smokers, but the difference also was not statistically significant. The possibility of a prolonged effect of smoking on the platelet aggregate ratio is more strongly suggested by the mean platelet aggregate ratios of 20 healthy habitual smokers and 20 healthy non-smokers of similar age and sex distribution. The mean platelet aggregate ratio of the 
smokers, who were asked to abstain from smoking for at least $8 \mathrm{hr}$ before determination of the platelet aggregate ratio, was 0.79 and of the non-smokers (Davis and Davis, 1980) was $0.91 \quad(P<0.05)$. The platelet aggregate ratios of patients with a variety of thromboembolic conditions have been reported to be low by most workers (Wu and Hoak, 1974, 1975; Wu et al., 1976; Gjesdal, 1976; Dougherty, Levy and Weksler, 1977; Guyton and Willerson, 1977; Wu, 1978; Mehta and Mehta, 1979; Salem et al., 1980) and normal by others (Prazich et al., 1977; Rohrer et al., 1978b). A lowered platelet aggregate ratio associated with the vascular diseases of the patients in this study may have tended to obscure a chronic effect of tobacco smoking on their ratios.

\section{Acknowledgment}

This study was supported by the Veterans Administration.

\section{References}

Carvalho, A.C.A., Colman, R.W. \& Lees, R.S. (1974) Platelet function in hyperlipoproteinemia. New England Journal of Medicine, 290, 434.

Chen, Y.C., Weiner, W.J., Walasek, J., SMith-McKearn, C. \& WU, K.K. (1979) Interrelationship of CPA, $\beta-T G$ and in vitro platelet aggregometry for the detection of platelet hyperaggregability. Blood, (Suppl. 1), 236a.

Davis, J.W. \& Davis, R.F. (1979) Acute effect of tobacco cigarette smoking on the platelet aggregate ratio. American Journal of the Medical Sciences, 278, 139.

Davis, J.W. \& Davis, R.F. (1980) Prevention of cigarette smoking-induced platelet aggregate formation by aspirin. Archives of Internal Medicine, 141, 206.

Dougherty Jr, J.H., Levy, D.E. \& WeksLeR, B.B. (1977) Platelet activation in acute cerebral ischaemia: serial measurements of platelet function in cerebrovascular disease. Lancet, i, 821.

Enger, S.C., Herbjørnsen, K., Erikssen, J. \& Fretland, A. (1977) High density lipoproteins (HDL) and physical activity: The influence of physical exercise, age and smoking on HDL-cholesterol and the HDL-/total cholesterol ratio. Scandinavian Journal of Clinical and Laboratory Investigation, 37, 251.

Erikssen, J., Hellem, A. \& Stormorken, H. (1977) Chronic effect of smoking on platelet count and 'platelet adhesiveness' in presumably healthy middle-aged men. Thrombosis and Haemostasis, 38, 606.

Gjesdal, K. (1976) Platelet function and plasma free fatty acids during acute myocardial infarction and severe angina pectoris. Scandinavian Journal of Haematology, 17, 205.
Guyton, J.R. \& Willerson, J.P. (1977) Peripheral venouß platelet aggregates in patients with unstable angina pecอ toris and acute myocardial infarction. Angiology, 28, 695C.

Kaplan, K.L., Broekman, M.J., Chernoff, A., Lesznik G.R. \& Drillings, M. (1979) Platelet $\alpha$-granule proteinso studies on release and subcellular localization. Blood, 53 604.

Karvonen, M., Orma, E., Keys, A., Fidanza, F. \& BrozeK J. (1959) Cigarette smoking, serum-cholesterol, blood $\frac{\text { }}{2}$ pressure, and body fatness: observations in Finland $\mathbb{Q}$ Lancet, i, 492.

Levine, S.P., Shuman, M.A., Raymond, N.M. \& KrentZ̋ L.S. (1979) The effect of exercise on measurements of in vivo platelet activation. Blood, (Suppl. 1), 250a.

Mehta, P. \& Mehta, J. (1979) Platelet function studies incoronary artery disease. V. Evidence for enhanced platelefw microthrombus formation activity in acute myocardias infarction. American Journal of Cardiology, 43, 757.

POZNER, H. \& BILlimoRIA, J.D. (1970) Effect of smoking op blood-clotting and lipid and lipoprotein levels. Lancet, ir 1318.

PraZiCh, J.A., RAPAPORT, S.I., SAMPles, J.R. \& Engler, R/ (1977) Platelet aggregate ratios - standardization of tech nique and test results in patients with myocardial ischaemia and patients with cerebrovascular disease. Thrombosis and Haemostasis, 38, 597.

Rohrer, T.F., Pfister, B., Weber, C., Imhof, P.R. \& STUCKI, P. (1978a) Validity of the Wu-Hoak method for the quantitative determination of platelet aggregatios in vivo. Blut, 36, 15.

Rohrer, T.F., Pfister, B., Weber, C., ImHof, P.R. \& STUCKI, P. (1978b) Quantitative changes in platelet aggregation due to physiological and pathological facforso and medication. Blut, 36, 21.

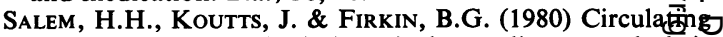
platelet aggregates in ischaemic heart disease and the correlation to platelet life span. Thrombosis Research, $17 \frac{\text { गे }}{\partial}$ 707.

Tномаs, C.B. (1958) Familial and epidemiologic aspects of coronary disease and hypertension. Journal of Chroniథ Diseases, 7, 198.

WU, K.K. (1978) Platelet hyperaggregability and thrombosi in patients with thrombocythemia. Annals of Internat Medicine, 88, 7.

Wu, K.K., Armstrong, M.L., Hoak, J.C. \& Megan, M. (1975) Platelet aggregates in hypercholesterolemic Rhesus. monkeys. Thrombosis Research, 7, 917.

Wu, K.K., BARNES, R.W. \& HoAK, J.C. (1976) Platelet hypere aggregability in idiopathic recurrent deep vein thrombosiso Circulation, 53, 687.

WU, K.K. \& HOAK, J.C. (1974) A new method for thg quantitative detection of platelet aggregates in patient? with arterial insufficiency. Lancet, ii, 924.

Wu, K.K. \& HoAK, J.C. (1975) Increased platelet aggree gates in patients with transient ischemic attacks. Stroke, $\mathbf{6}_{2}$ 521. 